\title{
Netzwerkmanagement und Bildung für eine nachhaltige Entwicklung
}

\section{Neue Schwerpunkte im kindheitspädagogischen Arbeitsfeld}

\author{
MICHAEL BRODOWSKI \\ Prof. Dr. phil. Michael Brodowski \\ lehrt an der Alice Salomon Hoch- \\ schule Berlin im Studiengang Erzie- \\ hung und Bildung im Kindesalter. \\ Seine Schwerpunkte sind Leitung \\ und Management frühkindlicher \\ Bildungseinrichtungen, Bildungspla- \\ nung und Bildungsmanagement. \\ brodowski@ash-berlin.eu
}

\author{
Die Entwicklung eines Masterstudiengangs über \\ Kindheitspädagogik ist ein guter Anlass, über den \\ Stand des Arbeitsfeldes nachzudenken. In zahlreichen \\ Gesprächen zwischen Theorie und Praxis zeichneten sich \\ dabei die beiden Schlagwörter "Netzwerkmanagement" \\ und "Bildung für eine nachhaltige Entwicklung" als \\ wichtige Aspekte für die Arbeit mit Kindern heraus.
}

Der Masterstudiengang an der Alice Salomon Hochschule Berlin (ASH) trägt den Arbeitstitel »Netzwerkmanagement Bildung für eine Nachhaltige Entwicklung - Schwerpunkt Kindheitspädagogik« und soll zum Sommersemester 2014 an den Start gehen. Das Interessante an der Entwicklung dieses Studienganges ist, dass diese mit über 90 Praxispartnern aus den Bereichen Kindheitspädagogik, Soziale Arbeit, Verwaltung, Berliner Senat, Kita-Trägern sowie Wirtschaftsunternehmen aus Berlin und Brandenburg umgesetzt wurde. So spiegelt sich in der fast zwei Jahre dauernden Entwicklungszeit, in der vier Workshops und mehrere korrigierende und ergänzende Evaluationsrunden stattfanden, unterschiedliche Ansichten über zukünftige Entwicklungen in einem kindheitspädagogischen Arbeitsfeld.

Dabei wurde zunächst gemeinsam mit den Praxispartnern an einem Verständnis dieses Masterstudienganges gearbeitet. So gibt es zwar eine stetig wachsende Zahl von Bachelor Studiengänge im Bereich der Kindheitspädagogik. Masterstudiengänge, die noch dazu maßgeblich durch die Praxis entwickelt wurden, sind dagegen seltener.

Welche Grundlage sollte gelegt werden? Wie sollte eine solche Qualifika- tion verstanden werden und vor allem was sagt die Praxis über die Art, wie dort gelernt werden soll?

Ein Masterstudium, so die beteiligten Akteure, baut auf bereits erworbenem Wissen, formal und informell erworbenen Fähigkeiten und Kompetenzen auf. Es muss also auch für berufsintegrierend und berufsbegleitend Studierende offen und in überschaubarer Zeit studierbar sein. Ein Masterstudium erkennt dieses Wissen, diese Fähigkeiten und Kompetenzen an und erweitert sie in einem gemeinsamen, wissenschaftlich fundierten Diskurs. So wird die Art des Lernens von hoher Selbstständigkeit geprägt und erlaubt es den Praxispartnern, diesen Master beispielsweise für ausgewählte Mitarbeitende ihres Unternehmens als hochwertige Personalentwicklungsmaßnahme zu begreifen.

Ein solches Masterstudium erlaubt es, selbst und mit anderen selbstständig zu lernen, $\mathrm{zu}$ arbeiten und eigene Schwerpunkte zu setzen. Es erfordert eine kritische und forschende Haltung, die nicht dem Selbstzweck dient, sondern eine fundierte und wissenschaftlich begründbare Einmischung in gesellschaftliche Entwicklungs- und Veränderungsprozesse verlangt. Somit sollen in einem solchen Masterstudium 
Studierenden, Lehrenden sowie Mentoren aus der Praxis als Einheit und aktive Gestalter eines kokonstruktiven, an Partizipation orientierten Lern- und Bildungsprozesses begriffen werden.

\section{Worum es geht}

In dem Masterstudiengang werden zukunftsorientiert und tragfähig die Querschnittsthemen Bildung für nachhaltige Entwicklung und Netzwerkmanagement im Schwerpunkt eines kindheitspädagogischen Arbeitsfeldes (0 bis 12 Jahre) behandelt. In das Konzept des Masters ist, neben wissenschaftlicher, forschungsorientierter Fundierung, ein starker Praxisbezug eingearbeitet. Konkrete, in übersichtliche Module gegliederte Handlungsfelder ermöglichen eine gute Orientierung sowie eine eigene, interessengeleitete Fokussierung durch die Möglichkeit, einen Schwerpunkt zu wählen. Dabei erfolgt die Unterstützung und Begleitung durch Mentorinnen und Mentoren aus der Praxis. Die Studierenden lernen Beteiligungsprozesse zu initiieren und mitzugestalten. Sie erhalten eine Qualifizierung in Hinblick auf und wie können sie gemeinsam, mit Trägern aus Ökonomie, Sozialem, Ökologie, Politik und Kultur umgesetzt werden?

Darüber hinaus steht die Entwicklung von pädagogischen Konzepten, die zielgruppenspezifische Ausgestaltung von Lern- und Bildungsorten sowie die Vernetzung von Akteuren im Mittelpunkt. Dabei wird dem Prinzip von Partizipation und Kooperation in Netzwerken gefolgt, die ebenso wesentliche Bestandteile einer Bildung für nachhaltige Entwicklung sind.

\section{Bildung für nachhaltige Entwicklung}

"Nachhaltige Entwicklung ist eine Entwicklung, die die Lebensqualität der gegenwärtigen Generation sichert und gleichzeitig zukünftigen Generationen die Wahlmöglichkeit zur Gestaltung ihres Lebens erhält", formulierte die Brundtlandkommission 1987. Nachhaltige Entwicklung hat vier Dimensionen: Ökonomische und ökologische, soziale und kulturelle Aspekte müssen

"Vernetztes Arbeiten ermöglicht

\section{Synergiepotenziale und konzeptionelle Weiterentwicklungen«}

Leitungstätigkeiten im mittleren und oberen Management Sozialer Träger, Quartieren aber auch der Arbeitsebene von Kommunen, Senaten und Ministerien. Dabei werden beispielsweise folgende Fragen exemplarisch in den Blick genommen:

- Wie leitet man kooperative Prozesse mehrerer Kitas im Sozialraum?

- Wie kann aus einem Quartier eine Bildungslandschaft für 0- bis 12-Jährige werden?

- Wie baut man bei einem Träger einen Bildungscampus auf, der eng mit dem sozialen Umfeld im Quartier zusammenarbeitet?

- Welche neuen Herausforderungen ergeben sich für die Kindheitspädagogik in Hinblick auf eine Bildung für eine nachhaltige Entwicklung dabei zusammenfließen (...). Im Sinne dieses Leitbilds ist es eine gesellschaftliche Aufgabe, die natürlichen Lebensgrundlagen für jetzige und folgende Generationen in der einen Welt zu bewahren und einen Beitrag zu sozialer Gerechtigkeit und kultureller Vielfalt $\mathrm{zu}$ leisten.

Bildung für nachhaltige Entwicklung (BNE) basiert auf der Erkenntnis, dass unsere derzeitige Art zu leben und zu wirtschaften nicht fortgesetzt werden kann, ohne dass ökologische Risiken sowie ökonomische und soziale Ungerechtigkeiten weltweit zunehmen. Der bereits spürbar stattfindende Klimawandel macht heute schon klar: Wir müssen uns deutlich und schnell umorientieren. Das gelingt nur, wenn viele Menschen gemeinsam im Sinne einer nachhaltigen Entwicklung handeln.
BNE möchte die Menschen dazu befähigen. Sie motiviert, Zukunftschancen $\mathrm{zu}$ erkennen und gemeinsam mit anderen aktiv und verantwortungsvoll $\mathrm{zu}$ nutzen. Zukunftsorientiertes Lernen und Handeln ist dabei an keine Altersstufe gebunden und richtet sich an alle Bildungsbereiche, schulische und außerschulische Lern- und Erfahrungsfelder - auch die der Früh- und Kindheitspädagogik.«(1)

\section{Bildung für nachhaltige Entwicklung in der Masterentwicklung}

Bildung für nachhaltige Entwicklung zieht sich als roter Faden durch die Gestaltung, Entwicklung und spätere Umsetzung des Masters.

\begin{abstract}
$1 \mathrm{Zu}$ betonen ist hier erstens der par- tizipative Grundgedanke sowie die Einbeziehung möglichst vieler Interessengruppen aus den Bereichen Umwelt, Wirtschaft, Sozialem, Politik und Verwaltung. Insgesamt 90 Interessierte aus diesen Bereichen arbeiteten an der Entwicklung des Masters mit.
\end{abstract}

Dies führt zum zweiten wesentli- chen Punkt, der Bewusstseinsbildung in Bezug auf Bildung für nachhaltige Entwicklung. Es gab und gibt nicht wenige Partner in den Arbeitsgruppen zur Masterentwicklung, die bisher mit Bildung für nachhaltige Entwicklung kaum in Berührung gekommen sind. In der Erarbeitung wesentlicher Elemente des Masters lernten sie von anderen viele unterschiedliche Perspektiven von Bildung für nachhaltige Entwicklung kennen, beschäftigten sich mit Konzepten einer Bildung für nachhaltige Entwicklung und setzten sich teilweise auch mit der möglichen Umsetzung von Bildung für nachhaltige Entwicklung Elementen in ihren Einrichtungen auseinander.

2 Der dritte wesentliche Punkt ist - die Überprüfung der einzelnen Elemente in Bezug auf Perspektiven der Entwicklungszusammenarbeit und mit dem Konzept der »einen Welt«. Auch hier lernten die Partner voneinander, wie dies in einzelnen Bereichen aufgehoben sein kann. So kann diese Perspektive etwa bei der Vernetzung von Kitas in Stadtteilen mit hohem Anteil von Kin- 
dern mit Migrationshintergrund eine wesentliche Rolle spielen.

Eine vierte Ebene des Bildungskon-zeptes ist es, Good Governance nicht nur $\mathrm{zu}$ thematisieren, sondern fest in der Konzeption des Masters zu verankern. Da die Studierenden später auch in der öffentlichen Verwaltung sowie den Arbeitsebenen von Ministerien und Senatsverwaltungen arbeiten sollen, musste der Master schon bei der Entwicklung wesentliche Felder dieses Ansatzes thematisieren und fest in den Modulen verankern.

Darüber hinaus wurde die Herangehensweise in der Masterentwicklung, bei der Gestaltung der Workshops und im Umgang der Partner untereinander von folgenden Punkten geprägt, die zu einem Bildung für nachhaltige Entwicklung Bildungskonzept zusammengezogen wurden: niemanden ausschließen, partizipativ arbeiten, zum Ausprobieren und Querdenken anregen, das eigene Handeln reflektieren, allen ein positives Lernen ermöglichen, die Entscheidungsfähigkeit der Partner fördern und schließlich Aspekte der nachhaltigen Entwicklung so transportieren, dass sie an die Erfahrungen der Einzelnen anschlussfähig sind.

\section{Bildung für eine nachhaltige Entwicklung in der Früh- und Kindheitspädagogik}

Die Deutsche UNESCO-Kommission weist explizit auf die Anknüpfungsfähigkeit des Bildungskonzeptes einer Bildung für nachhaltige Entwicklung an frühkindliche Bildung (2) hin. Sie appelliert an verantwortliche Akteure, Bildung für nachhaltige Entwicklung als entscheidendes Qualitätsmerkmal in die frühkindliche Bildung zu integrieren. (3) Das Bild vom Kind, wie es im Masterstudiengang verstanden wird, entspricht den vier Grundsätzen, welche dem Bachelorstudiengang Erziehung und Bildung im Kindesalter zugrunde liegen (4):

1 Das Kind ist von der ersten Lebens- minute an bestrebt, sich seine Wirklichkeit aus eigener Initiative und mit eigenen Mitteln anzueignen, mit seiner Umwelt in Kontakt zu treten und Erfahrungen zu sammeln.

Kinder sind Subjekte ihres eigenen - Bildungsprozesses, die in der Auseinandersetzung mit der Umwelt Sinn und Bedeutung suchen.

3 Jedes Kind ist ein soziales Wesen, 3. das mit anderen Menschen in Kontakt tritt, Beziehungen sucht und benötigt. Im sozialen Austausch und durch die Fürsorge seiner engsten Bezugspersonen entwickelt es tiefgehende Bin-

\section{Neuer Masterstudiengang zur Kindheitspädagogik}

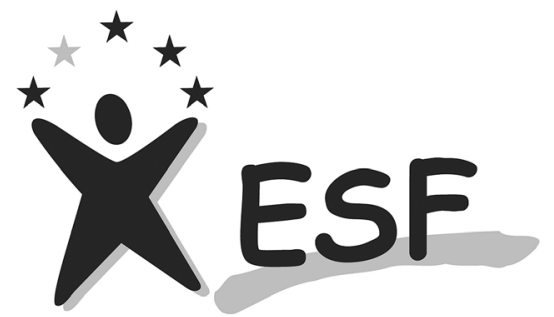

...eine Chance durch Europa!

Der Masterstudiengang mit dem Arbeitstitel "Netzwerkmanagement Bildung für eine Nachhaltige Entwicklung - Schwerpunkt Kindheitspädagogik" an der Alice Salomon Hochschule Berlin (ASH) soll zum Sommersemester 2014 beginnen. Die Entwicklung eines solchen Masterstudienganges, die partizipativ und maßgeblich von Praxispartnern gestaltet wird, wirkt auf das kindheitspädagogische Arbeitsfeld zurück.

Zum einen werden hier wesentliche Kompetenzen aus unterschiedlichen Perspektiven von Trägern und Partnern zusammengetragen: Was soll eine Absolventin können und in welchen Bereichen sollte sie sich auskennen. Diese Grundfrage beschäftigte die Partner in den Workshops immer wieder. Was also sind auch die Defizite im Arbeitsfeld der Kindheitspädagogik, wo wissen Absolventen bisher zu wenig, welche innovativen Felder will die Praxis zukünftig besetzen und ausbauen.
Eine solche Entwicklung zeigt zum anderen, dass eine Bestandsaufname nötig ist. Sie zeigt, dass manches schon vorhanden, aber nicht genug bekannt ist. Sie zeigt, wo es Reibungsverluste in der zukünftigen Zusammenarbeit von Hochschule, Senatsverwaltung und Trägern der Sozialen Arbeit und Kindheitspädagogik gibt, die man im Rahmen eines solchen Studiums immer wieder neu thematisieren und bearbeiten kann. Daher präferierten die Träger für den Master Netzwerkmanagement als Methode und Bildung für eine nachhaltige Entwicklung als innovativen Inhalt.

Der Masterstudiengang wurde gemeinsam mit der Leuchtpol gGmbH und über 90 Praxispartnern sowie einem bundesweiten Expertenbeirat entwickelt und von E.ON AG finanziell gefördert. Diese partizipative Entwicklung wurde von der Deutschen Unesco Kommission im September 2012 mit der Auszeichnung: "Offizielles Projekt der UN Weltdekade Bildung für eine Nachhaltige Entwicklung" geehrt. Aktuell wird die Etablierungsphase bis zum 31. Dezember 2013 vom Europäischen Sozialfonds Berlin gefördert.

Mehr Informationen zu dem neuen Studiengang: Prof. Dr. Michael Brodowski, Alice Salomon Hochschule Berlin, Leitung und Management frühkindlicher Bildungseinrichtungen, E-Mail brodowski@ash-berlin. eu, Telefon 030 99245-209.

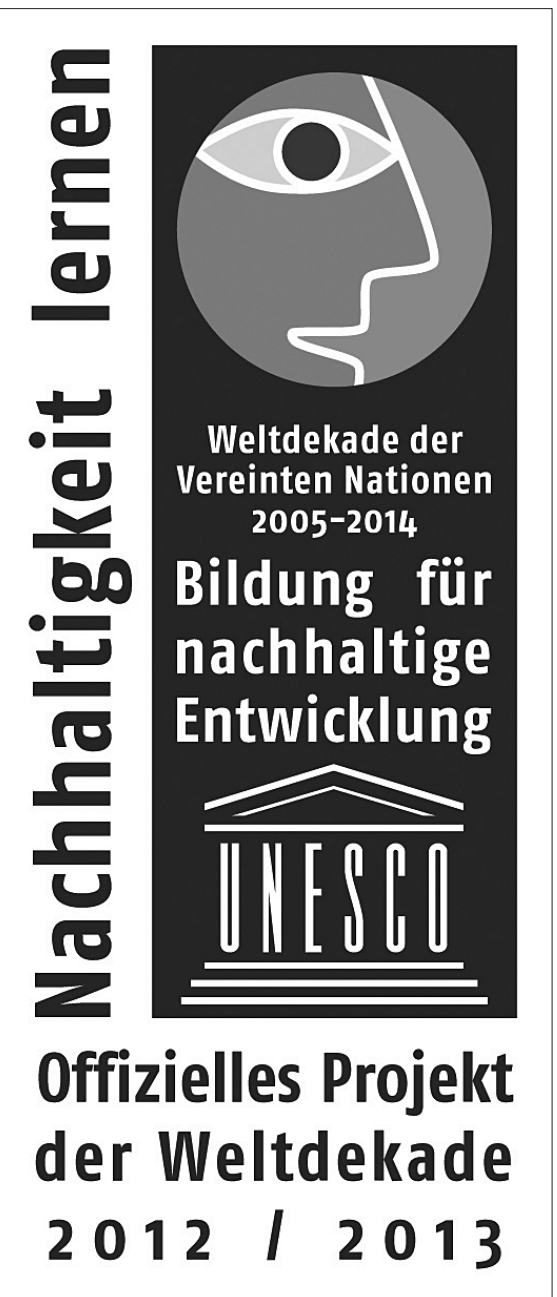


dungsbeziehungen, die eine bedeutsame Basis für sein eigenaktives Welterkunden darstellen und deren Qualität die kindliche Selbst- und Weltkonstruktion erheblich beeinflusst.

1 Jedes Kind hat ungeachtet seines - Geschlechts, seiner Herkunft, Religion, Lebensweise, seines Alters und Entwicklungsstandes das Recht, in seiner Individualität ernst genommen und wertgeschätzt zu werden.

\section{Netzwerke sind kein Selbstzweck}

Oft wird mit Netzwerken die Hoffnung verbunden, soziale Belange und Belange einer nachhaltigen Entwicklung besser durchsetzen zu können und Unterstützung für die Betroffenen und Aktiven schnell und unbürokratisch zu leisten.

So kann ein Netzwerk zur "Nachbarschaftshilfe " in einem Stadtteil sehr effektiv und direkt helfen, in dem man sich beispielsweise auf unterschiedliche Weise und unentgeltlich - nur auf Gegenleistung beruhend - damit unterstützt, was man am Besten kann. (5) Damit geht auch die Hoffnung einher, dass man in Netzwerken mehr Gemeinschaftlichkeit und Menschlichkeit erlebt, als es beispielsweise in Arbeitsorganisationen der Fall ist. Alle Partner sind nahezu gleichberechtigt und arbeiten das zur Gemeinschaft zu, was sie am Besten können. Damit verbindet sich häufig der hehre Anspruch einer nicht entfremdeten Arbeit und menschlicher Formen der Zusammenarbeit.

Auch können durch Vernetzung Synergiepotenziale im Zuge der Zusammenarbeit steigen. Wenn zwei Kitas mit anderen freien Trägern und Grundschulen zusammenarbeiten, könnten sie mit dem gleichen oder sogar weniger Ressourceneinsatz gemeinsam und partizipativ mehr Möglichkeiten für alle schaffen. Dabei lernen die Teilnehmenden in diesem Netzwerk voneinander und entwickeln sich im Idealfall gemeinsam weiter.

Bildung für nachhaltige Entwicklung lebt in und von solchen Netzwerken. Von über eintausend ausgezeichneten Projekten zur Bildung für nachhaltige Entwicklung (siehe www. bne-portal.de) gibt es kaum eines, das nicht mit Partnern zusammengearbeitet hat!
Netzwerke sind aber kein Selbstzweck, man kann sich nicht in ihnen verstecken oder ausruhen. Die Mitgliedschaft ist oft an direkte Beteiligung gebunden, die mehr eigene Investitionen verlangt, als man sie ursprünglich kalkuliert hat. Netzwerke zu pflegen und zu erhalten kann einen hohen Koordinationsaufwand erfordern.

Daher ist es wichtig, ihren Aufbau anzuregen und zu unterstützen, sie zu managen und ihren Erhalt zu sichern. Es ist wichtig, fördernde und hemmende Rahmenbedingungen für die Netzwerke zu erforschen, sie zu kennen und in diese Rahmenbedingungen gestaltend einzugreifen. Das erfordert, mit Blick auf eine nachhaltige Entwicklung das koordinierte Zusammenwirken von Ökonomie, Ökologie, Sozialem, Politik und Kultur.

\section{Anmerkungen}

(1) Aus: Leuchtpol gemeinnützige Gesellschaft zur Förderung von Umweltbildung im Elementarbereich mbH (2009): Leuchtpol - Ein Projekt zur Förderung von Bildung für nachhaltige Entwicklung im Kindergarten.

(2) Bildung für nachhaltige Entwicklung in der Masterentwicklung.

(3) Deutsche UNESCO-Kommission e. V. (Hg.) (2010): Zukunftsfähigkeit im Kindergarten vermitteln: Kinder stärken, nachhaltige Entwicklung befördern.

(4) Aus ASH (2011): Modulhandbuch Bachelor Studiengang. Erziehung und Bildung im Kindesalter (berufsintegrierende Studienform).

(5) DJI (Hg.) (2004): Konzepte und Strategien der Netzwerkarbeit. Reader DJI der Tagung vom 13. bis 15.Oktober 2004.

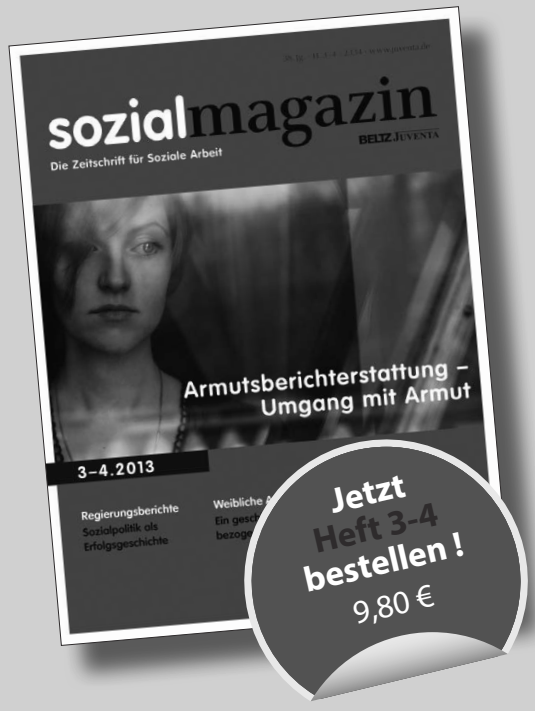

Thema Heft 3-4/2013

Armutsberichterstattung Umgang mit Armut

- Regierungsberichte Sozialpolitik als Erfolgsgeschichte

- Berichterstattung über Arme oder über Armut?

- Weibliche Armut Ein geschlechtsbezogenes Risiko

- Arbeiten in Unsicherheit Arm trotz Arbeit

- Alternative Wege - Das bedingungslose Grundeinkommen und seine Folgen

- Verwundbarkeit und Erschöpfung. Konsequenzen für die Armutsberichterstattung

Preis Heft 3-4/2013: € 9,80 zzgl. Versandkosten

\section{Bestellen Sie Heft 3-4 hier}

Telefon 06201/6007-330

Fax 06201/6007-9331

E-Mail: medienservice@beltz.de Internet: www.juventa.de Beltz Medienservice

Postfach 100565

D-69445 Weinheim

BELZJUVENTA 